\title{
Decoding of human hand actions to handle missing limbs in neuroprosthetics
}

\author{
Jovana J. Belić ${ }^{1,2+}$ and A. Aldo Faisal ${ }^{1,3,4 *}$ \\ ${ }^{1}$ Department of Bioengineering, Imperial College London, London, UK \\ 2 Faculty of Electrical Engineering, University of Belgrade, Belgrade, Serbia \\ ${ }^{3}$ Department of Computing, Imperial College London, London, UK \\ ${ }^{4}$ Integrative Biology, MRC Clinical Sciences Centre, Faculty of Medicine, Imperial College London, London, UK
}

\section{Edited by:}

Martin Giese, University Clinic

Tübingen, Germany

\section{Reviewed by:}

Rolf Pfeifer, University of Zurich, Switzerland

Kai Xu, Shanghai Jiao Tong

University, China

*Correspondence:

A. Aldo Faisal, Brain and Behaviour Laboratory, Royal School of Mines

Building, Imperial College London,

SW7 2AZ London, UK

e-mail: a.faisal@imperial.ac.uk

${ }^{\dagger}$ Present address:

Jovana J. Belić, Department of Computational Biology, Royal Institute of Technology KTH, Stockholm, Sweden
The only way we can interact with the world is through movements, and our primary interactions are via the hands, thus any loss of hand function has immediate impact on our quality of life. However, to date it has not been systematically assessed how coordination in the hand's joints affects every day actions. This is important for two fundamental reasons. Firstly, to understand the representations and computations underlying motor control "in-the-wild" situations, and secondly to develop smarter controllers for prosthetic hands that have the same functionality as natural limbs. In this work we exploit the correlation structure of our hand and finger movements in daily-life. The novelty of our idea is that instead of averaging variability out, we take the view that the structure of variability may contain valuable information about the task being performed. We asked seven subjects to interact in 17 daily-life situations, and quantified behavior in a principled manner using CyberGlove body sensor networks that, after accurate calibration, track all major joints of the hand. Our key findings are: (1) We confirmed that hand control in daily-life tasks is very low-dimensional, with four to five dimensions being sufficient to explain $80-90 \%$ of the variability in the natural movement data. (2) We established a universally applicable measure of manipulative complexity that allowed us to measure and compare limb movements across tasks. We used Bayesian latent variable models to model the low-dimensional structure of finger joint angles in natural actions. (3) This allowed us to build a naïve classifier that within the first $1000 \mathrm{~ms}$ of action initiation (from a flat hand start configuration) predicted which of the 17 actions was going to be executed-enabling us to reliably predict the action intention from very short-time-scale initial data, further revealing the foreseeable nature of hand movements for control of neuroprosthetics and tele operation purposes. (4) Using the Expectation-Maximization algorithm on our latent variable model permitted us to reconstruct with high accuracy $\left(<5-6^{\circ} \mathrm{MAE}\right)$ the movement trajectory of missing fingers by simply tracking the remaining fingers. Overall, our results suggest the hypothesis that specific hand actions are orchestrated by the brain in such a way that in the natural tasks of daily-life there is sufficient redundancy and predictability to be directly exploitable for neuroprosthetics.

Keywords: neurotechnology, motor control, neuroprosthetics, movement variability, Bayesian classifier, activities of daily living, finger movement

\section{INTRODUCTION}

The human hand is a highly complex actuator and perhaps the most important and diverse tool we use to interact with the environment. The hand is capable of both a powerful grip to push, pull, or twist objects, and a precise grip to twist and turn small objects or handles (Napier, 1980). These are just a few of the countless gestures we can use and learn. Anatomically, the hand comprises a total of 27 bones, 18 joints, and 39 muscles (Tubiana, 1981), which afford over 20 degrees of freedom (DOF) (Stockwell, 1981; Soechting and Flanders, 1997; Jones and Lederman, 2006). The number of degrees of freedom is an important characterization of the human hand because it defines the dimensionality of the control problem that has to be solved by the motor system. However, previous studies of human motor control showed that normal hand behavior uses only a small subset of possible hand configurations (Todorov and Ghahramani, 2004; Weiss and Flanders, 2004; Ingram et al., 2008; ValeroCuevas et al., 2009). It is known that biomechanically, the control of individual joints is limited by the redundant set of muscles that control single or several joints (Lang and Schieber, 2004; Rácz et al., 2012). Studies of neural and neuromuscular architecture of the hand have demonstrated that these do not support fully 
isolated joint movements (Lemon, 1997; Poliakov and Schieber, 1999; Reilly and Schieber, 2003), and biomechanical constraints appear to result in all muscles being required for full directional control of grip forces (Kutch and Valero-Cuevas, 2011). Additionally, it has been proposed that motor control of the hand joints is organized in a modular way, where several degrees of freedom are organized into functional groups to simplify the control problem (Santello et al., 1998; Tresch et al., 2006).

In the realm of muscle co-actions so called motor synergies were identified to represent structured spatio-temporal patterns of muscle interplay in defined movements (Bernstein, 1967; Santello et al., 2002; Daffertshofer et al., 2004; d'Avella et al., 2006; Tresch et al., 2006). Also, studies that have focused on finger joint kinematics of complex hand shapes (Santello et al., 1998; Mason et al., 2001; Daffertshofer et al., 2004), as well as continuous daily-life-day activity (Ingram et al., 2008) found that most variability in the data could be explained by just a few (four to six) characteristic parameters (so called principal components) that indicates a high degree of correlation between the angles of the fingers. These have also been replicated in studies focusing on the key evolutionary ability to produce flint-stone tools (Faisal et al., 2010).

The importance of the hand as our means to interact with the world becomes painfully evident when loss of a hand or hand function occurs. Here neuroprosthetics and robotic hands have rapidly evolved to imitate an unprecedented level of hand-control (Wolpaw and McFarland, 1994, 2004; Taylor et al., 2002; Bitzer and van der Smagt, 2006; Carrozza et al., 2006; Hochberg et al., 2006, 2012; Kuiken et al., 2007, 2009; Rothling et al., 2007; Steffen et al., 2007; Zhou et al., 2007; Cipriani et al., 2008; Liu et al., 2008; Velliste et al., 2008; Schack and Ritter, 2009; Schröder et al., 2012; Feix et al., 2013; Thomik et al., 2013). Yet, it is still very difficult for people with a lost limb to achieve naturalistic mobility and dexterity by controlling a prosthetic replacement in the same way they would control their own body. This increases the training time to use such neuroprosthetics (up to 2 years) and results in a low adoption rate after training.

We hypothesize that natural hand movements performed "inthe-wild," outside artificially construed and highly controlled laboratory tasks contain correlation information that can be used for prediction and reconstruction in the context of prosthetics. We asked subjects to perform everyday tasks such as opening the door, eating, using the phone, etc. The data consists of 15dimensional time series representing the angles of all the major joints of all the fingers. Advances in experimental methods have increased the availability, amount and quality of high-resolution behavioral data for both humans and animals that can be collected. However, most behavioral studies lack adequate quantitative methods to model behavior and its variability in a natural manner. Here, we take the view that motor behavior can be understood by identifying simplicity in the structure of the data, which may reflect upon the underlying control mechanisms. Yet, the analysis of movements and specifically hand movements is complicated by the highly variable nature of behavior (Faisal et al., 2008). To extract the structure of hand configuration variability data stream we used a probabilistic generative latent variable model (PPCA) of hand configurations for each task.
Part of these results was previously published in the form of abstracts (Belić and Faisal, 2011, 2014).

\section{MATERIALS AND METHODS SUBJECTS}

Seven adults (two women and five men, average age $24 \pm 2$ years) with no known history of neurological or musculoskeletal problems, participated in this study following approved ethical guidelines. All subjects were right-handed as determined by the Edinburgh Handedness Inventory (Oldfield, 1971). The experimental procedure used in this experiment was approved by the local ethics committee.

\section{EXPERIMENTS AND DATA ACQUISITION}

We asked subjects to perform 17 different everyday tasks (Figure 1), while capturing their hand movements by using resistive sensors embedded in a previously calibrated CyberGlove I (CyberGlove System LLC, CA, USA). The data glove is made of thin cloth, and its sensors are correlated with corresponding joints of the human hand (Figure 2A). The CyberGlove we used in this study is associated with 18 DOF of the hand. We used data from 15 sensors that consisted of metacarpalphalangeal (MCP) and proximal interphalangeal (PIP) sensors for the four fingers, three stretch sensors between the four fingers, three sensors for the thumb (the carpometacarpal (CMC), MCP and interphalangeal (IP) sensors), and the stretch sensor between the thumb and the palm of the hand. Sensors were sampled continuously at $80 \mathrm{~Hz}$ at a resolution of eight bits per sensor. Subjects completed 10 repetitions for each of the activities, and they always started trials from the same initial position (the hand was placed on the interface device attached to the subject's belt with the fingers composed together and thumb oriented parallel to the palm). The beginning of each trial was indicated with a sound. The trials were self-paced and the purpose of activities was explained to subjects orally, but they were not instructed about any desired movements for the upcoming trials. After performing the task, the subject then returned his/her hand to the initial position. All programs for data acquisition, visualization and calibration were purpose-developed in $\mathrm{C}++$.

\section{CALIBRATION}

The output of each CyberGlove sensor is voltage value (raw value) which is dependent on the bending applied to that specific sensor. In order to obtain the outputs in degrees (Figure 2B), it is necessary to determine conversion factor gain and a constant term offset for each of the sensors. This process is called calibration of the CyberGlove. Once the gain and offset are set, output in degrees of the corresponding sensor is given by the following equation:

$$
\text { angle }=\text { gain } *(\text { RawValue }- \text { offset })
$$

To calculate the gain and offset we need two different pre-defined angles for each of the sensors and raw values that correspond to them (RawValue ${ }_{1}$ and RawValue 2 ). Gain and offset are calculated by the following formulas: 

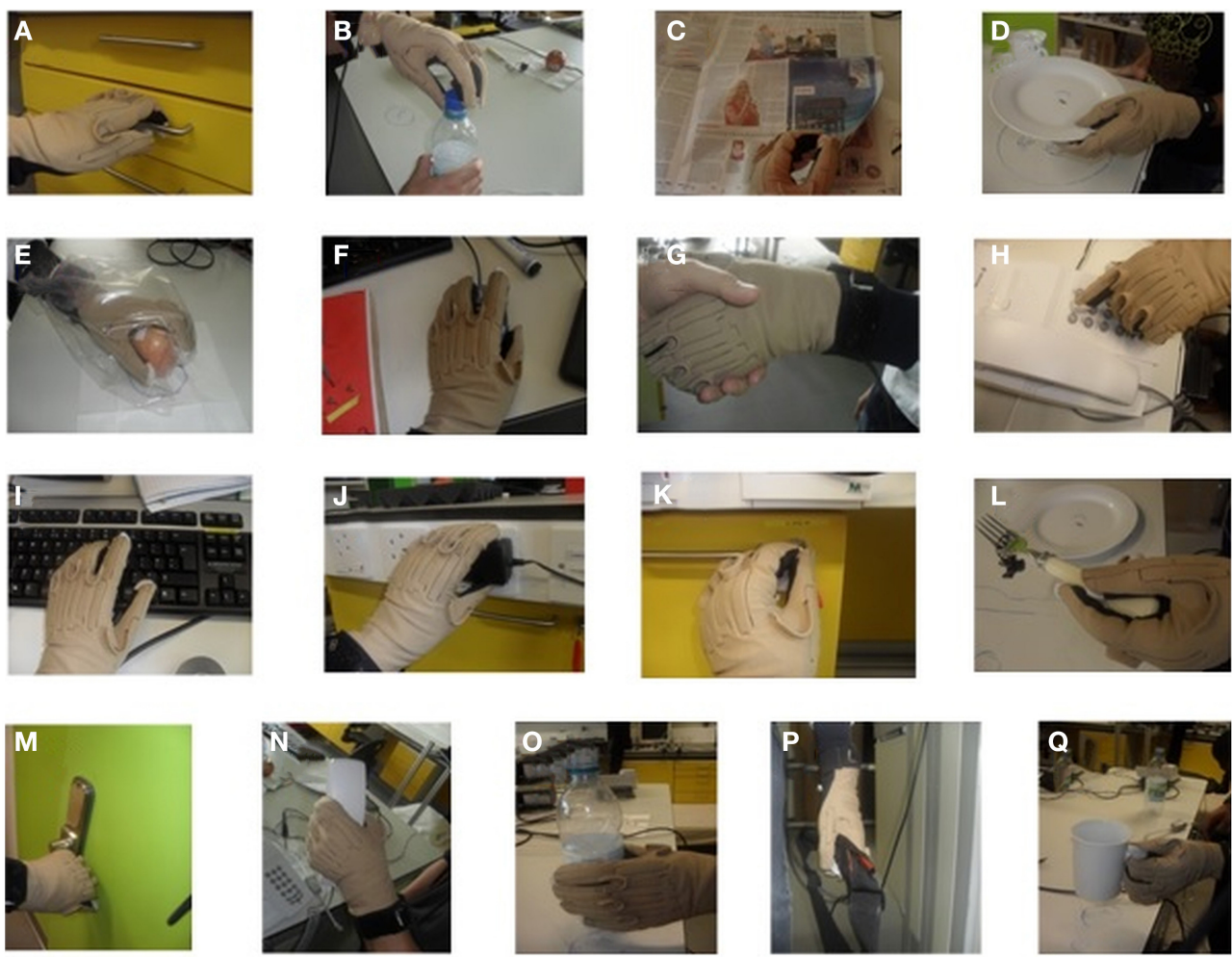

FIGURE 1 | Subjects were involved in 17 different everyday activities. (A) Opening and closing a drawer. (B) Removing a bottle cap (unwinding and winding). (C) Turning the pages of a newspaper (one page in each trial). (D) Picking up a plate, putting it on the marked location, and returning it to the starting position. (E) Eating an apple (subject takes one bite of the apple) and returning the apple to the starting position. (F) Manipulating a mouse in a pre-defined way. (G) Handshaking for a duration of $5 \mathrm{~s}$. (H) Dialling pre-defined numbers on telephone. (I) Typing pre-defined text on a keyboard. (J) Manipulating a plug and returning it to the starting position. (K) Opening a door using a key and returning the key to the starting position. (L) Picking up and putting down an object using a fork. (M) Opening and closing a door using the knob. (N) Picking up a telephone handle. (O) Picking up a plastic bottle, simulating drinking, and returning the bottle to the starting position. (P) Picking up and putting down a bag. (Q) Picking up a glass with a handle, simulating drinking, and returning the glass to the starting position.
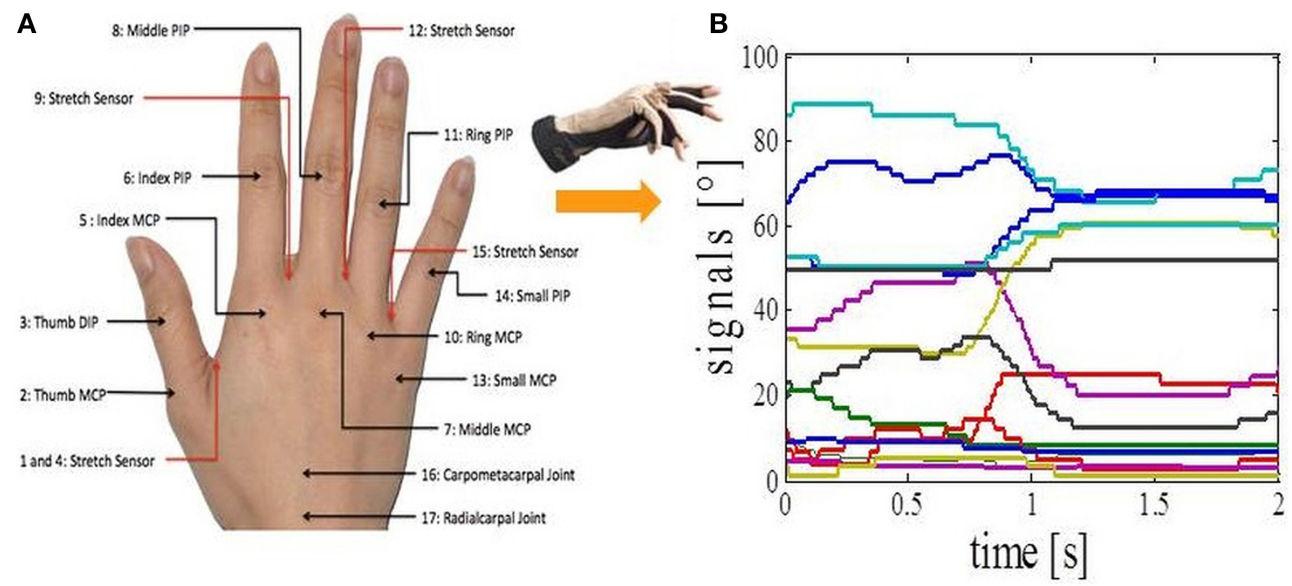

FIGURE 2 | Data acquisition. (A) CyberGlove sensor locations. (B) Calibrated output signals from the CyberGlove for one of the activities. 


$$
\begin{array}{r}
\text { gain }=\frac{\left(\text { angle }_{1}-\text { angle }_{2}\right)}{\left(\text { RowValue }_{1}-\text { Row Value }_{2}\right)}, \\
\text { offset }=\text { RowValue }_{1}-\frac{\text { angle }_{1}}{\text { gain }} .
\end{array}
$$

The glove was calibrated for each subject using a five-step procedure that allowed us to determine two different angles (angle $e_{1}$ and angle $e_{2}$ ) for each of the sensors (Figure 3):

The first position corresponded to $0^{\circ}$ for all glove sensors (Figure 3A).

The second position defined an angle of $90^{\circ}$ for all MCP sensors except for the thumb (Figure 3B).

The third position determined the abduction angles of $30^{\circ}$ between the middle and index finger and between the little and ring finger, an angle of $20^{\circ}$ between the ring and middle finger, and an angle of $90^{\circ}$ between the index and thumb finger.

The fourth position defined an angle of $90^{\circ}$ for all PIP sensors except for the thumb.

The fifth position corresponded to the angles for the thumb sensors: $\mathrm{CMC}\left(90^{\circ}\right), \mathrm{MCP}\left(45^{\circ}\right)$, and IP $\left(90^{\circ}\right)$ sensor.

The calibration procedure was further improved using an online visualization system. In our study, a virtual human hand was rendered in OpenGL. The virtual hand was animated in real-time by data from the glove (Figure 3F). Visualization of data was of great help during both calibration and data acquisition processes. In the case of visually observed deviation between the 3D model and the actual position of the hand, gain and offset were re-determined only for the sensors where deviation was observed. Calibration parameters for each of the subjects had been stored in a separate file and loaded before the experiments started. We also asked subjects, after completing the calibration procedure, to again place their hand in the first position, so we could additionally check eventual discrepancies. The average error across the sensors was 5 \pm 2 degrees.

\section{COMPUTATIONAL LATENT VARIABLE MODELING OF REAL-LIFE MOVEMENTS}

Collected data from the 15 sensors for each subject and each trial were stored to disk for offline analysis using MatLab [MathWorks, Natick (MA)]. Before further analysis, the data is smoothed using a second-order Savitzky-Golay filter with a running window of five data points to remove discontinuities induced by the $\mathrm{A} / \mathrm{D}$ converter.

Our data space potentially extends over a 15-dimensional space. We performed Principle Component Analysis (PCA) on joint angles in order to estimate real dimensionality of the finger movements during daily activities. PCA reduces the set of correlated variables to a set of non-correlated variables (principle components) (Semmlow, 2001; Bishop, 2006). The first principal component contains as much of the variability (as quantified by the variance) in the data as possible, as does each succeeding component for the remaining variability. Therefore, here we used the PCA method to determine the complexity of the finger movements, by measuring how many principal components can explain most of the variability in the data (Faisal et al., 2010). For example, dimensionality reduction techniques can be illustrated by considering the index finger, which has three joints controlled by five muscles. Describing the flexing behavior of this finger requires a priori three values ("dimensions"). For example, in specific movements like making a fist, as we flex one joint of the index finger, we flex the other two joints at the same time in a highly coordinated manner. Thus, we would require in principle a single dimension to describe the configuration of the finger. PCA ignores the temporal structure of movements (in fact the results of PCA will be the same if the data in each trial is randomly shuffled in time). Thus, correct classification relies on the sub-space of finger movement variability alone.

Tipping and Bishop found a probabilistic formulation of PCA by viewing it as a latent variable problem, in which a d-dimensional observed data vector $\boldsymbol{x}$ can be described in terms of an m dimensional latent vector, $y$ :

$$
x=W y+\mu+\epsilon,
$$
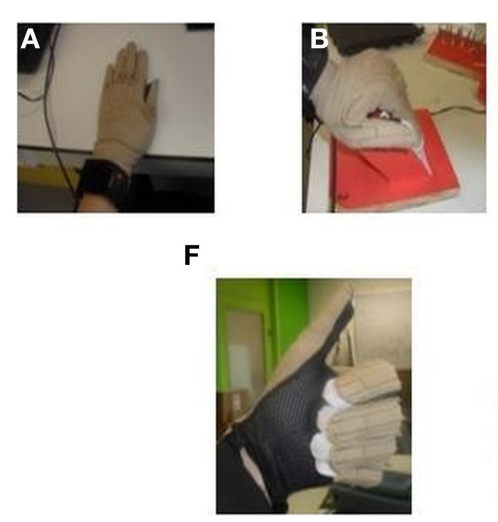
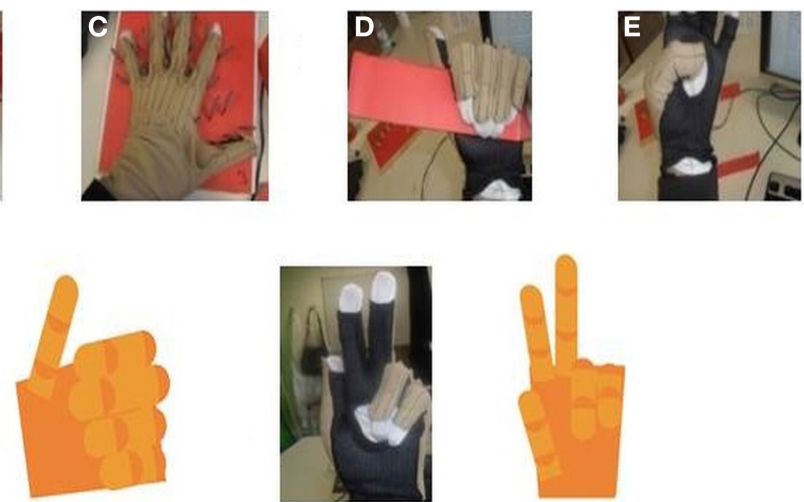

FIGURE 3 | CyberGlove calibration procedures. (A) The position defines angle $_{1}$ for all CyberGlove sensors. (B) The position defines angle 2 for sensors that correspond to MCP joints of the four fingers. (C) The position defines $a^{a n g l e} e_{2}$ for abduction sensors. (D) The position defines angle ${ }_{2}$ for sensors that correspond to PIP joints of the four fingers. (E) The position defines angle 2 for sensors that were used to measure the position of the thumb. (F) Examples for real time capturing of finger movements by using a 3D hand model that was developed to further improve accuracy of the calibration procedure. 
where $W$ is $d \times m$ matrix, $\mu$ is the data mean and $\epsilon$ is an independent Gaussian noise with a diagonal covariance matrix $I$. The likelihood of observed data vector $\boldsymbol{x}$ is given as:

$$
p(\boldsymbol{x})=(2 \pi)^{-d / 2}|\boldsymbol{C}|^{-1 / 2} e^{\left(-1 / 2(\boldsymbol{x}-\boldsymbol{\mu})^{T} \boldsymbol{C}^{-1}(\boldsymbol{x}-\boldsymbol{\mu})\right)},
$$

and $\operatorname{Cov}$ is the model covariance matrix given by the following formula:

$$
\boldsymbol{C o v}=\boldsymbol{W} \boldsymbol{W}^{T}+\sigma^{2} \boldsymbol{I} .
$$

$W$ and $\sigma$ are obtained by iterative maximization of log-likelihood of $p$ :

$$
\begin{gathered}
\sigma^{2}=\frac{1}{d-m} \sum_{k=m+1}^{d} \gamma_{k}, \\
\boldsymbol{W}=\boldsymbol{U}_{m}\left(\boldsymbol{A}_{m}-\sigma^{2} \boldsymbol{I}\right)^{\frac{1}{2}} \boldsymbol{R},
\end{gathered}
$$

where $\gamma_{k}$ are eigen values, $\boldsymbol{U}_{m}$ is $d \times m$ matrix of eigenvectors, $\boldsymbol{A}_{m}$ is diagonal matrix $(m \times m)$ of eigen values, and $\boldsymbol{R}$ is an arbitrary $m \times m$ orthogonal rotation matrix (for simplicity $\boldsymbol{R}$ is usually equal to $I$ ).

\section{MEASURE OF MANIPULATIVE COMPLEXITY}

As a way to quantify manipulative complexity for a given number of PCs, we proposed a universally applicable measure that allowed us to calculate and compare limb movements across different tasks. We refer to it as manipulative complexity $C$, and define the measure by the following formula:

$$
C=1-\frac{2}{N-1} \sum_{j=1}^{N} \sum_{i=1}^{j}\left(\text { Variance explained by } P C_{i}-1 / N\right)
$$

where $N$ is the total number of PCs we consider. Our data space extends over a 15-dimensional space, so if all PCs contribute equally that implies $C=1$, and $C=0$ if one PC explains all data variability. Our complexity measure compares well-with intuitive complexity estimates and it can be thought of as a new assessment measure that is calculated after an objective mathematical analysis. For example, a simple behavior, e.g., curling and uncurling a hand into a fist, would reveal a single dominant principal component as all five fingers (and each finger's joint) move in a highly correlated manner and therefore $C$ would be close to 0 . In contrast, a complex behavior, such as expert typing on a keyboard would reflect more uniform distribution of variances explained by principal components, as each finger moves independently from the others, and so $C$ would have a high value.

\section{TASK RECOGNITION FROM MOVEMENT DATA (BAYESIAN CLASSIFICATION)}

Next, we simply predicted a task based on the one with the highest PPCA likelihood by employing Bayesian classifier. In Bayesian statistics there are two important quantities: unobserved parameters $\Omega_{j}(j=1, \ldots, 17$ different activities in our study) and observed data $\boldsymbol{x}$ (movement data). They are related in the following way:

$$
P\left(\Omega_{j} \mid \boldsymbol{x}\right)=\frac{P\left(\boldsymbol{x} \mid \Omega_{j}\right) P\left(\Omega_{j}\right)}{P(\boldsymbol{x})},
$$

where $P\left(\Omega_{j} \mid \boldsymbol{x}\right)$, which is termed posterior, represents probability that testing data $\boldsymbol{x}$ belong to activity $\Omega_{j}$. Prior, $P\left(\Omega_{j}\right)$, is simply given by the relative frequency of occurrence of each class in the training set and we can ignore it here. Therefore, probability of each class, given testing data, is equal to likelihood $P\left(x \mid \Omega_{j}\right)$ (probability of seeing the data given the task) that is thoroughly explained in Section Computational Latent Variable Modeling of Real-life Movements.

For training and testing the classifier we used leaveone-repetition (across all actions and all subjects)-out crossvalidation.

\section{MISSING LIMB MOVEMENT RECONSTRUCTION (LATENT VARIABLE DECODING)}

For data reconstruction, firstly we used linear regression to fit the data of missing joints as a function of other joints and expressed results as the average difference between actual and predicted values. Then, we employed the Expectation-Maximization (EM) algorithm for PPCA in order to estimate missing values and at the same time to determine the right subspace dimension. In the EM approach for PPCA, we considered the latent variables $y_{n}$ to be "missing" data and the "complete" data to encompass the observations together with these latent variables (Tipping and Bishop, 1999). The corresponding complete-data log-likelihood is given as:

$$
\begin{aligned}
L c & =\sum_{n=1}^{N} \ln \left(\mathrm{p}\left(x_{\mathrm{n}}, \boldsymbol{y}_{\mathrm{n}}\right)\right), \\
p\left(\boldsymbol{x}_{n}, \boldsymbol{y}_{n}\right) & =\left(2 \pi \sigma^{2}\right)^{-d / 2} e^{\left(-\frac{\left\|x_{n}-W y_{n}-\mu\right\|^{2}}{2 \sigma^{2}}\right)(2 \pi)^{-m / 2}} e^{-\frac{\left\|y_{n}\right\|^{2}}{2}} .
\end{aligned}
$$

Then we calculated the expectation (E-step) of $L_{C}$ :

$$
\begin{aligned}
\langle L c\rangle= & -\sum_{n=1}^{N}\left\{\frac{d}{2} \ln \left(\sigma^{2}\right)+\frac{1}{2} \operatorname{tr}\left(\left\langle\mathbf{y}_{n} \mathbf{y}_{n}^{t}\right\rangle\right)+\frac{1}{2 \sigma^{2}}\left(\mathbf{x}_{n}-\mu\right)^{t}\left(\mathbf{x}_{n}-\mu\right)\right. \\
& \left.-\frac{1}{\sigma^{2}}\left\langle\mathbf{y}_{n}\right\rangle^{t} \mathbf{W}^{t}\left(\mathbf{x}_{n}-\mu\right)+\frac{1}{2 \sigma^{2}} \operatorname{tr}\left(\mathbf{W}^{t} \mathbf{W}\left\langle\mathbf{y}_{n} \mathbf{y}_{n}^{t}\right\rangle\right)\right\}, \text { where } \\
\left\langle\boldsymbol{y}_{n}\right\rangle= & C^{-1} \mathbf{W}^{t}\left(\boldsymbol{x}_{n}-\boldsymbol{\mu}\right), \\
\left\langle\boldsymbol{y}_{n} \boldsymbol{y}_{n}^{t}\right\rangle= & \sigma^{2} \boldsymbol{C}^{-1}+\left\langle\boldsymbol{y}_{n}\right\rangle\left\langle\boldsymbol{y}_{n}\right\rangle^{t} .
\end{aligned}
$$

In the M-step, $L_{C}$ was maximized with respect to $\boldsymbol{W}$ and $\sigma^{2}$ :

$$
\begin{aligned}
& \tilde{\boldsymbol{W}}=\left[\sum_{n}\left(\boldsymbol{x}_{n}-\boldsymbol{\mu}\right)\left\langle\boldsymbol{y}_{n}\right\rangle^{t}\right]\left[\sum_{n}\left\langle\boldsymbol{y}_{n} \boldsymbol{y}_{n}^{t}\right\rangle\right]^{-1} \\
& \tilde{\sigma}^{2}=\frac{1}{N d} \sum_{n=1}^{N}\left\{\left\|\boldsymbol{x}_{n}-\boldsymbol{\mu}\right\|^{2}-2\left\langle\boldsymbol{y}_{n}\right\rangle^{t} \tilde{\boldsymbol{W}}^{t}\left(\boldsymbol{x}_{n}-\boldsymbol{\mu}\right)+\operatorname{tr}\left(\left\langle\boldsymbol{y}_{n} \boldsymbol{y}_{n}^{t}\right\rangle \tilde{\boldsymbol{W}}^{t} \widetilde{\boldsymbol{W}}\right)\right\}
\end{aligned}
$$


These equations were iterated until the algorithm was judged to have converged.

\section{RESULTS}

\section{NATURAL HAND AND FINGER JOINT KINEMATICS HAVE A LOW-DIMENSIONAL EMBEDDING}

The structure of natural hand and finger movements in dailylife is characterized by a highly variable nature. Even in the case of handshaking (Figure 1G), which represents one of the most stereotypic actions, basic statistical analysis has revealed vast diversity in angular data for MCP and PIP joints across different subjects (Figure 4A). In this work we first exploited the correlations between MCP and PIP joints for each of the four fingers and we found that correlation coefficients were stronger for little and ring fingers and weaker for middle and index fingers (Figure 4B). Further, correlations between each of the four fingers were highest for the neighboring fingers and gradually decreased for more distant fingers (Figure 4C). We also used Principal Component Analysis in order to estimate dimensionality of the finger movements across different complex manipulation tasks. Therefore, we used PCA as a measure for the complexity of hand configuration, by measuring the amount of variance in the data displayed by each of the principal components. For example, a simple behavior such as curling and uncurling the hand would reveal a single dominant PC component, as all finger joints move in a highly correlated manner. In Figure 5A we show the percentage of explained variance vs. the number of used principal components for each of the 17 activities. PCA revealed for all tasks that hand motor control restricted hand configurations on a low dimensional subspace of four to five dimensions (which explained $83-96 \%$ of the variance in the data), in line with previous data on evolutionary relevant hand behavior (crafting of flint stone tools, Faisal et al., 2010) and non-annotated long-term statistics of joint velocities (Ingram et al., 2008). These results imply a substantial reduction from the 15 degrees of freedom that were recorded. Some of the activities required more principal components than others to reconstruct the data. For example in Figure 5A we can see that opening a lid on a bottle or manipulating a fork are far more complex activities than hand dialling numbers on a phone. Single PC component explained around $30 \%$ less variance in the first case (opening a lid) than in the second (dialling numbers), while that discrepancy was around $10 \%$ in the case when we used only the first four PCs to explain variance.

\section{MEASURING THE MANIPULATIVE COMPLEXITY OF ACTIVITIES IN DAILY LIFE}

We can visually observe some differences and similarities in the manipulative complexity between the most simple hand movements, during which the individual joints move in a highly correlated manner, and the most complex, where each finger moves independently from the others. Here we proposed a universally applicable measure of manipulative complexity $(C)$ that allows us

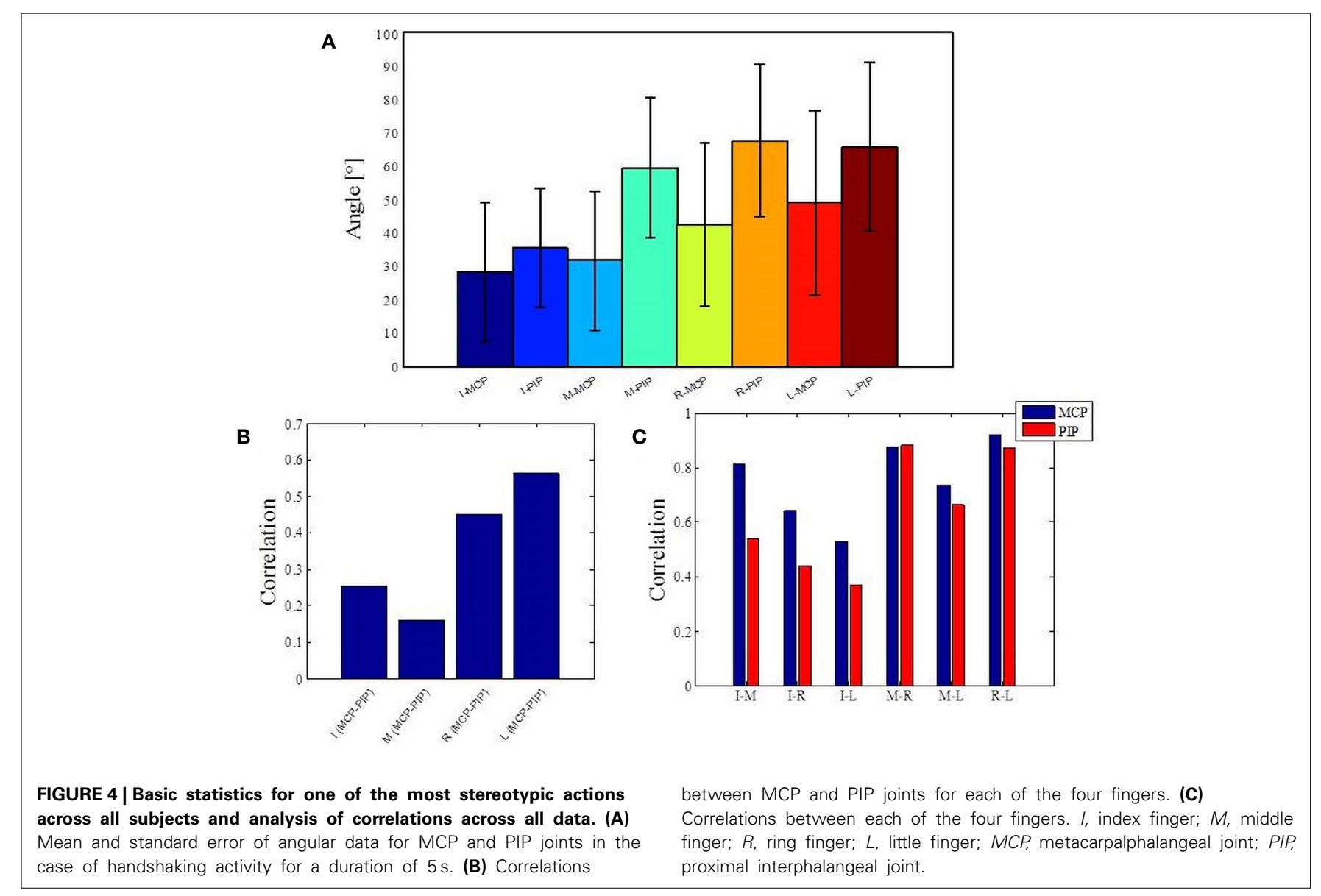




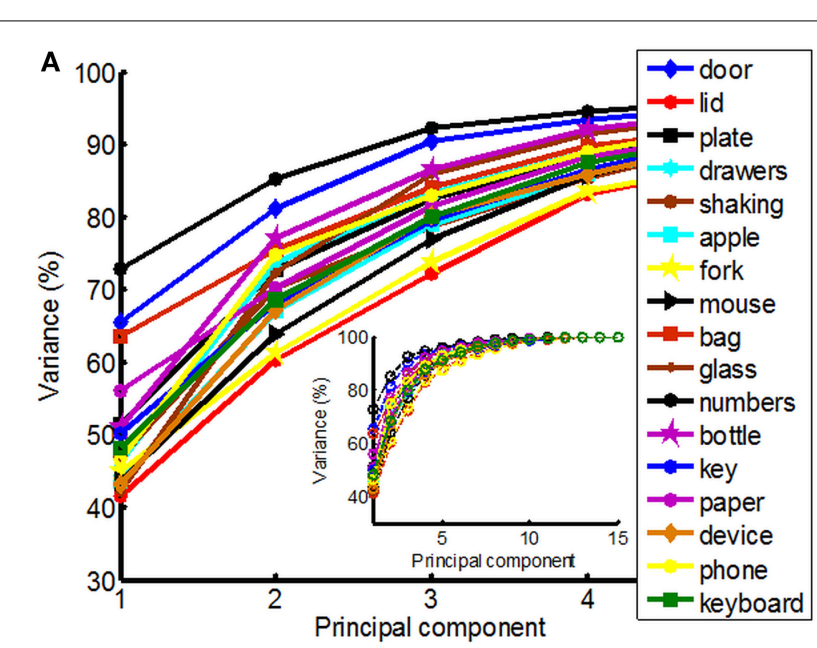

B

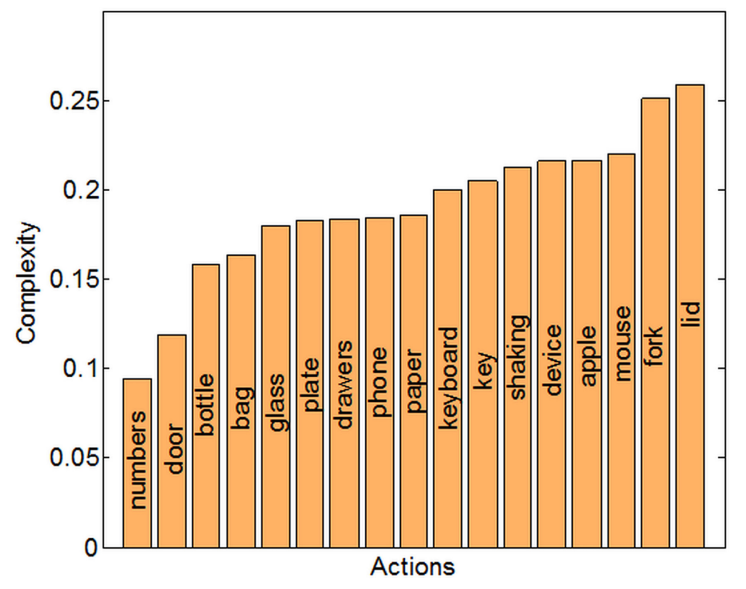

FIGURE 5 | Principal component analysis (PCA) and quantitative measure of manipulative complexity. (A) Curves show the cumulative sum of variance (expressed in percentage) explained by increasing the numbers of principal components separately for each of the 17 daily-life activities. The $x$-axis corresponds to the number of PCs; the $y$-axis shows the percentage of the variance of the finger movements explained by the respective number of PCs. (B) Our proposed quantitative measure of manipulative complexity (manipulative complexity is maximal (equal to 1 ) if all DOF contribute equally, and minimal (equal to 0 ) if one DOF explains all DOF).

to measure this quantity across vastly different tasks. Our complexity measure implies that $C=1$ if all DOF contribute equally (the most complex activities), and $C=0$ if one DOF explains all DOF (the most simple activities). Results produced are in line with intuitive expectations (opening a lid on a bottle is more complex than operating a door handle) (Figure 5B). Some of the activities in our study also included "grasp like motions" (e.g., operating a door handle, grabbing a bottle or grabbing a bag) that visually would look very similar. Our established complexity measure appeared sensitive enough and was able to differentiate between even those similar looking grasps.

\section{PREDICTION OF HAND MOVEMENTS FROM INITIAL MOVEMENT DATA}

Further, we wanted to see how different subspaces influence success of classification for different tasks. To deal with this, we

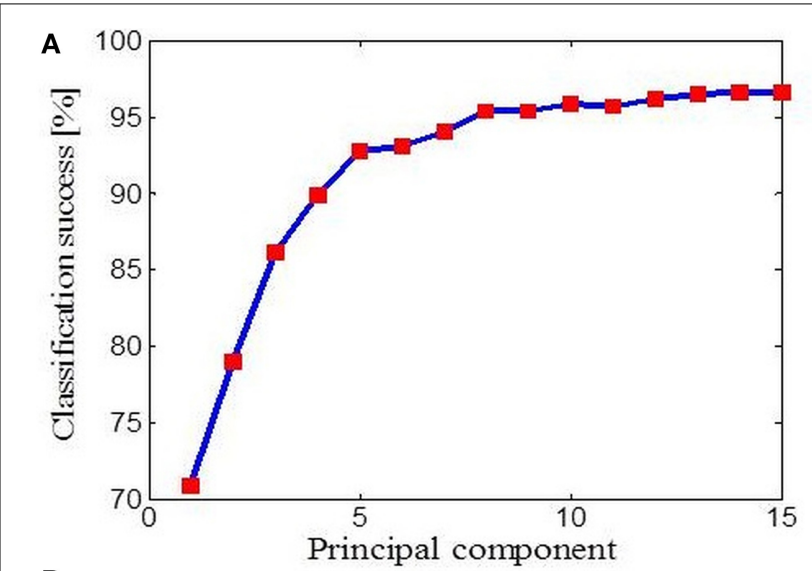

B

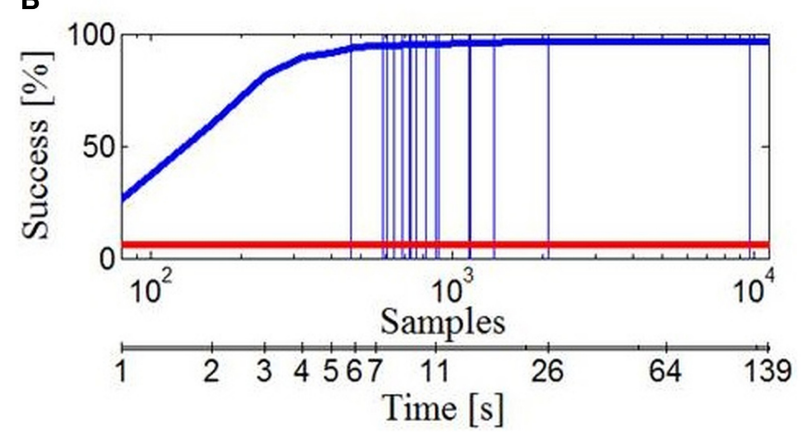

FIGURE 6 | Data classification. (A) Classification performance with reference to the number of PPCA components. (B) Classification performance with reference to the number of data samples taken (duration of activity). Performance by chance is marked with the red line and vertical lines represent the average number of data samples (duration) for each of 17 activities.

used Bayesian PPCA. PPCA has been considered as a mechanism for probabilistic dimension reduction or as a variable-complexity predictive density model (Tipping and Bishop, 1999) and correct classification relies on the subspace of finger movement variability alone. Figure 6A illustrates the success of classification with reference to the number of PPCA components. Therefore, by using only the first few PPCA components in the classification process we can get very high classification success. For example, using the first four PPCA components the success of classification was 89.91\% (across all tasks, classification performance was $96.63 \%$ using all 15 PPCA components). Importantly, in Figure 5 one could see that extracted subspaces appeared to be task-dependant, which suggests that besides simplification, synergies might have a role in a task-optimal control as well. If specific tasks can engage specific motor control strategies, then we should be able to make a conclusion regarding the task by observing some early portions of finger data. Indeed, the classification performance, presented in Figure 6B, was a few times higher than the chance performance (marked with red line) for only an initial portion of the finger configuration samples of each task. Within the first $1000 \mathrm{~ms}$ from the initial hand position, which was identical for every action, it appeared that hand shape already configures itself to a specific task and we were able to quickly predict intended action. Vertical lines represent average duration for each of 17 activities. 


\section{RECONSTRUCTION OF MISSING LIMBS' MOVEMENTS BY DECODING MOVEMENTS OF REMAINING LIMBS}

Next, we investigated the predictability of a subset of joint movements in respect of the movements of other joints. Or in other words, if part of a limb is missing, how well-can we predict what those missing parts should be doing by only observing the intact, remaining limb parts. This is of fundamental interest in prosthetic control. We focused particularly on cases where data had been acquired with sensors that measure the bending around the MCP or the PIP joints of the four missing fingers. First, we applied linear regression in the case of missing values from the MCP joints (Figure 7A) and the PIP joints (Figure 7B) for each of the four fingers separately. The error we got, measured as absolute difference between predicted and actual joint values and averaged across all tasks, showed the best linear predictability for the middle and ring fingers in both examined cases. Overall predictability rate was high regarding movement range $\left(90^{\circ}\right)$ for each of the considered joints and variability of tasks. Then, we applied an EM algorithm for PPCA to infer the un-observed, invisible joints in the case of missing data from the MCP sensors. Figure 7C shows obtained results with reference to the number of PPCA components. Here the best results were also acquired for the middle and ring fingers. The error was the highest in the case when just one PPCA component was used and then started to decrease (up to a number around eight PPCA). Generally, these results could help us to improve the method of designing prosthetic controllers that are driven by intact limb parts and support neuroprosthetic controllers in refining the decoding of action intention of users.

\section{DISCUSSION}

We analyzed natural movements from the seven subjects who were behaving spontaneously while performing 17 different everyday activities. We have four key findings that we will discuss individually in more detail as follows: (1) Regarding activities of daily living, we confirmed that hand control is low-dimensional, i.e., four to five PCs explained $80-90 \%$ of the variability in the movement data. (2) We established a universally applicable measure of manipulative complexity that allowed us to measure this quantity across vastly different tasks. Our findings are in line with intuitive expectations (opening a lid on a bottle is more complex than hand dialling numbers) and are sensitive enough to
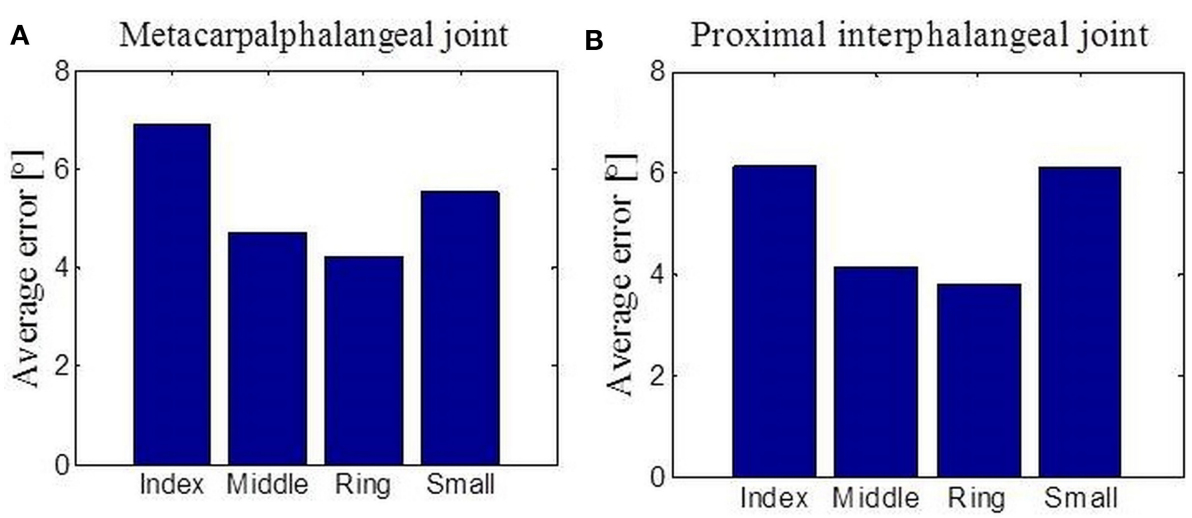

C

Metacarpalphalangeal joint

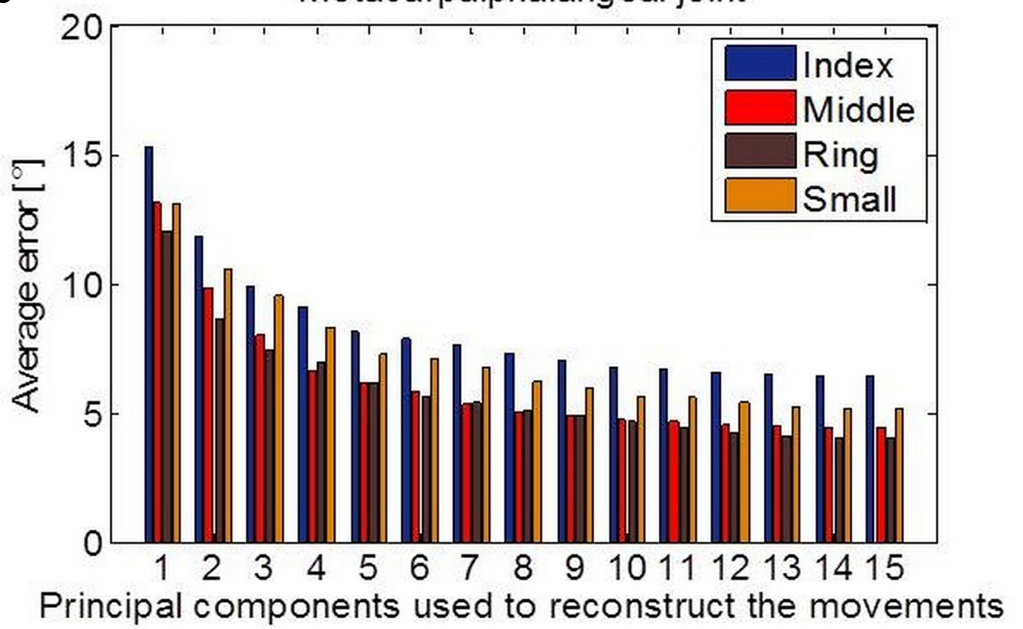

FIGURE 7 | Data reconstruction. (A) Average error after linear reconstruction in the case when data from MCP sensors of the four fingers were missing. (B) Average error after linear reconstruction in the case when data from PIP sensors of the four fingers were missing. (C) Results of data reconstruction by using PPCA with reference to the different number of PC components used. 
differentiate between similar looking interactions (e.g., operating a door handle is less complex than grabbing a bottle). (3) We discovered that within the first $1000 \mathrm{~ms}$ of an action the hand shape already configures itself to vastly different tasks, enabling us to reliably predict the action intention. (4) We suggest how the statistics of natural finger movements paired with Bayesian latent variable model can be used to infer the movements of missing limbs from the movements of the existing limbs to control for example, a prosthetic device.

In many everyday activities we move our fingers in a highly correlated manner. Therefore, it has been proposed that control of human hand movements is organized in a way that comprises coupling of several DOF into functional groups. The opinion that motor synergies lie behind manual actions has been supported by several studies (Santello et al., 1998, 2002; Daffertshofer et al., 2004; d'Avella et al., 2006; Tresch et al., 2006; Ingram et al., 2008; Faisal et al., 2010; Jarrassé et al., 2014). The most common interpretation is in terms of simplifying the strategy that the central nervous system might undertake. Studies that have investigated hand configurations during reaching and grasping movements (Santello et al., 1998; Mason et al., 2001) reported that $90 \%$ of the variance in hand configurations could be explained by only three principal components. In our study PCA analysis revealed that in 17 daily activities hand configurations operated on lowdimensional subspace (four to five dimensions) as well, which is also in line with previous data on evolutionary relevant hand behavior (crafting of flintstone tools) (Faisal et al., 2010) and nonannotated long-term statistics of joint velocities (Ingram et al., 2008). These finding supports the view that the motor cortex organizes behavior in a low-dimensional manner to avoid the curse of dimensionality in terms of computational complexity. We also found numerical differences in the number of principle components required to explain a given amount of variability in hand configurations across each of the tasks. Similar conclusions were obtained in the case of a small number of much simpler manipulation tasks (Todorov and Ghahramani, 2004; Bläsing et al., 2013).

Our manipulative complexity measure, established for the first time, gave us a chance to quantify the complexity of the movements across a high number of different activities. This was very important in that some of the activities that look highly similar (grasp like motions such as operating a door handle, grabbing a bottle or grabbing a bag) apparently had different values of complexity. Those findings demonstrated also that our complexity measure is sensitive enough to differentiate between similar looking interactions. The highest value of complexity had tasks of opening a lid on a bottle or manipulating a fork, and the lowest had tasks of dialling numbers on a phone or opening a door using the door knob. Results produced are in line with intuitive expectations regarding the fact that in the first two cases one is expected to have high engagement of the thumb that is the most individuated (Häger-Ross and Schieber, 2000). In the case of typing numbers, most of the subjects used their index finger while their other fingers created some form of fist, and in case of opening the door our fingers move in a highly correlated manner. Here we compare structures of complex dynamic hand manipulations, while some other studies (Feix et al., 2009, 2013) have presented a successful methodology for measuring and evaluating the capability of artificial hands to produce 31 different human-like grasp postures.

Further we employed Bayesian PPCA on the behavioral data in order to analyze the structure of variability within it. Variability is ubiquitous in the nervous system and it has been observed in actions even when external conditions are kept constant (Harris and Wolpert, 1998; Faisal et al., 2008). In this paper we take the view that the hand configuration variability may contain significant information about the task being performed. Our approach yields an effective assessment of the tasks that subjects were involved with. The Bayesian PPCA reveals that the finger movement correlations are so structured that we can obtain very high classification success by taking only first few principal components. Regarding motor control, it has been suggested that structural learning (Braun et al., 2009a,b) may reduce the dimensionality of the control problem that the learning organism has to search in order to adapt to a new task. Our results are in line with this concept and suggest the hypothesis that the brain can engage many sets of motor controllers, which are selected based on specific tasks, and which also orchestrate resulting actions in overall behavior and produce movement variability in characteristic sub-spaces. Next we thought that, if the hypothesis is true, we should be able to infer the task the hand is engaged in by observing some initial portion of the finger movement data. Crucially, observing only the initial portion of hand configurations (from our identical starting position) was sufficient to characterize the entire hand task, and the classification performance we obtained was a few times higher than chance performance.

A common approach in design of neuroprosthetics is to construct body parts that can be controlled with the same functionality as natural limbs. Using a reduced set of basic functions to construct internal neural representation could be essential from an optimal control perspective (Poggio and Bizzi, 2004) and applied to neuroprosthetics control (Thomik et al., 2013). Our linear predictability of the missing joints based on movements of other finger joints gave good results. The best results were achieved for middle and ring fingers showing that they are the least individuated. This is in line with the previous research (Häger-Ross and Schieber, 2000). Further, the PPCA algorithm for missing data revealed that using more than eight PPCA components does not lead to any significant improvement. In this study we perform action recognition and reconstruction of missing finger trajectories using the current positions of other functional finger joints by simply requiring - in principle - the user to act out with his functional fingers an intended task. Such finger motion can be realized with cheap wearable wireless sensors (Gavriel and Faisal, 2013) and we can reconstruct the natural behavior of users without the need for expensive, training intensive, noninvasive or invasive electrophysiological interfaces. Consequently, unlike common approaches that require the user to learn to use the technology, the technology interprets the natural behavior of users (Abbott and Faisal, 2011). Thus, the neuronal and biomechanically imposed correlation structure of hand-finger can be exploited to build smart, sensitive neuroprosthetics controllers that infer the task "at hand" based on the movements of the remaining joints. 
Dexterous object manipulation is conditioned by the continuous interactions between the body and the environment and engages multiple sensory systems. Vison can provide essential information for controlling hand kinematics in the cases when object are fully visible. Human manipulation involves also tactile signals from different types of mechanoreceptors in the hand that allows humans to easily hold a very wide range of objects with different properties without crushing or dropping them (Johansson and Flanagan, 2009). Tactile sensing provides also critical information in avoiding slipping as crucial precondition to successfully manipulate an object, what is most apparent in people with impaired tactile signals. When finger contact with the desired object is made, we start to increase the grasp force to the optimal level, using both our prior knowledge about the object and information from the tactile sensors of the fingers gathered during the interaction (Johansson and Flanagan, 2008; Romano et al., 2011). Corrective actions are applied to different frictional conditions in order to provide an optimal grip force that is normally $10-40 \%$ greater than the minimum required to prevent slips (Johansson and Flanagan, 2008). Consequently, future neuroprosthetics should provide reliable user's intention decoding as well as optimal sensory feedback (Berg et al., 2013; Raspopovic et al., 2014). Therefore, looking into hand kinematics as an important aspect of the hand capabilities represents just one approach that forms the basis for future studies. Further inclusion of other parameters that are of relevance and investigating their influence on manipulative complexity will provide a more complete analysis.

\section{ACKNOWLEDGMENTS}

JJB was supported by the IASTE scholarship of the British Council. AAF acknowledges the support of the Human Frontiers Program Grant (HFSP RPG00022/2012).

\section{REFERENCES}

Abbott, W., and Faisal, A. (2011). Ultra-low cost eyetracking as an highinformation-throughput alternative to BMIs. BMC Neurosci. 12(Suppl. 1):P103. doi: 10.1186/1471-2202-12-S1-P103

Belić, J. J., and Faisal, A. A. (2011). The structured variability of finger motor coordination in daily tasks. BMC Neurosci. 12(Suppl. 1):P102. doi: 10.1186/14712202-12-S1-P102

Belić, J. J., and Faisal, A. A. (2014). Bayesian Approach to Handle Missing Limbs in Neuroprosthetics. Götingen: Bernstein conference Götingen.

Berg, J. A., Dammann, J. F., Tenore, F. V., Tabot, G. A., Boback, J. L., Manfredi, L. R., et al. (2013). Behavioral demonstration of a somatosensory neuroprosthesis. IEEE Trans. Neural Syst. Rehabil. Eng. 21, 500-507. doi: 10.1109/TNSRE.2013.2244616

Bernstein, N. A. (1967). The Co-ordination and Regulation of Movements. Oxford: Pergamon Press.

Bishop, C. (2006). Pattern Recognition and Machine Learning. New York, NY: Springer Science+Business Media, LLC.

Bitzer, S., and van der Smagt, P. (2006). "Learning EMG control of a robotic hand: towards active prostheses," in Proceedings of the IEEE International Conference on Robotics and Automation (ICRA 2006) (Orlando, FL), 2819-2823. doi: 10.1109/ROBOT.2006.1642128

Bläsing, B., Maycock, J., Bockemühl, T., Ritter, H., and Schack, T. (2013). Motor synergies and mental representations of grasping movements," in ICRA 2013 Workshop on Hand synergies - How to Tame the Complexity of Grasping (Karlsruhe).

Braun, D. A., Aertsen, A., Wolpert, D. M., and Mehring, C. (2009a). Motor task variation induces structural learning. Curr. Biol. 19, 352-357. doi: 10.1016/j.cub.2009.01.036
Braun, D. A., Mehring, C., and Wolpert, D. M. (2009b). Structure learning in actions. Behav. Brain Res. 206, 157-165. doi: 10.1016/j.bbr.2009.08.031

Carrozza, M. C., Cappiello, G., Micera, S., Edin, B. B., Beccai, L., and Cipriani, C. (2006). Design of a cybernetic hand for perception and action. Biol. Cybern. 95, 629-644. doi: 10.1007/s00422-006-0124-2

Cipriani, C., Zaccone, F., Micera, S., and Carrozza, M. C. (2008). On the shared control of an EMG-controlled prosthetic hand: analysis of user-prosthesis interaction. IEEE Trans. Robot. 24, 170-184. doi: 10.1109/TRO.2007.910708

Daffertshofer, A., Lamoth, C. J., Meijer, O. G., and Beek, P. J. (2004). PCA in studying coordination and ariability: a tutorial. Clin. Biomech. 19, 415-428. doi: 10.1016/j.clinbiomech.2004.01.005

d'Avella, A., Portone, A., Fernandez, L., and Lacquaniti, F. (2006). Control of fast-reaching movements by muscle synergy combinations. J. Neurosci. 26, 7791-7810. doi: 10.1523/JNEUROSCI.0830-06.2006

Faisal, A., Selen, L., and Wolpert, D. (2008). Noise in the nervous system. Nat. Rev. Neurosci. 9, 292-303. doi: 10.1038/nrn2258

Faisal, A., Stout, D., Apel, J., and Bradley, B. (2010). The manipulative complexity of lower paleolithic stone toolmaking. PloS ONE 5:e13718. doi: 10.1371/journal.pone.0013718

Feix, T., Pawlik, R., Schmiedmayer, H., Romero, J., and Kragic, D. (2009). "A comprehensive grasp taxonomy," in Robotics, Science and Systems: Workshop on Understanding the Human Hand for Advancing Robotic Manipulation (Seattle, WA), 2-3.

Feix, T., Romero, J., Ek, C. H., Schmiedmayer, H. B., and Kragic, D. (2013). A Metric for comparing the anthropomorphic motion capability of artificial hands. IEEE Trans. Robot. 29, 82-93. doi: 10.1109/TRO.2012.2217675

Gavriel, C., and Faisal, A. A. (2013). Wireless kinematic body sensor network for low-cost neurotechnology applications "in-the-wild." IEEE/EMBS Conf. Proc. Neural Eng. 6, 1279-1282. doi: 10.1109/NER.2013.6696174

Häger-Ross, C., and Schieber, M. H. (2000). Quantifying the independence of human finger movements: comparisons of digits, hands, and movement frequencies. J. Neurosci. 20, 8542-8550.

Harris, C. M., and Wolpert, D. M. (1998). Signal-dependent noise determines motor planning. Nature 394, 780-784. doi: 10.1038/29528

Hochberg, L. R., Bacher, D., and Jarosiewicz, B. (2012). Reach and grasp by people with tetraplegia using a neurally controlled robotic arm. Nature 485, 372-375. doi: 10.1038/nature11076

Hochberg, L. R., Serruya, M. D., Friehs, G. M., Mukand, J. A., Saleh, M., Caplan, A. H., et al. (2006). Neural ensemble control of prosthetic devices by a human with tetraplegia. Nature 442, 164-171. doi: 10.1038/nature04970

Ingram, J., Körding, K., Howard, I., and Wolpert, D. (2008). The statistics of natural hand movements. Exp. Brain Res. 188, 223-236. doi: 10.1007/s00221-0081355-3

Jarrassé, N., Ribeiro, A. T., Sahbani, A., Bachta, W., and Roby-Brami, A. (2014). Analysis of hand synergies in healthy subjects during bimanual manipulation of various objects. J. Neuroeng. Rehabil. 11, 1-11. doi: 10.1186/1743-000311-113

Johansson, R. S., and Flanagan, J. R. (2008). "Tactile sensory control of object manipulation in humans," in The Senses: a Comprehensive Reference 6, eds E. Gardner and J. H. Kaas (San Diego, CA: Academic), 67-86. doi: 10.1016/B978012370880-9.00346-7

Johansson, R. S., and Flanagan, J. R. (2009). Coding and use of tactile signals from the fingertips in object manipulation tasks. Nat. Rev. Neurosci. 10, 345-359. doi: $10.1038 /$ nrn2621

Jones, L. A., and Lederman, S. J. (2006). Human Hand Function. Oxford: Oxford University Press. doi: 10.1093/acprof:oso/9780195173154.001.0001

Kuiken, T. A., Li, G., Lock, B. A., Lipschutz, R. D., Miller, L. A., Stubblefield, K. A., et al. (2009). Targeted muscle reinnervation for realtime myoelectric control of multifunction artificial arms. JAMA 301, 619-628. doi: 10.1001/jama.2009.116

Kuiken, T. A., Miller, L. A., Lipschutz, R. D., Lock, B. A., Stubblefield, K., Marasco, P. D., et al. (2007). Targeted reinnervation for enhanced prosthetic arm function in a woman with a proximal amputation: a case study. Lancet 369, 371-380. doi: 10.1016/S0140-6736(07)60193-7

Kutch, J. J., and Valero-Cuevas, F. J. (2011). Muscle redundancy does not imply robustness to muscle dysfunction. J. Biomech. 44, 1264-1270. doi: 10.1016/j.jbiomech.2011.02.014

Lang, C. E., and Schieber, M. H. (2004). Human finger independence: limitations due to passive mechanical coupling versus active neuromuscular control. J. Neurophysiol. 92, 2802-2810. doi: 10.1152/jn.00480.2004 
Lemon, R. N. (1997). Mechanisms of cortical control of hand function. Neuroscientist 3, 389-398. doi: 10.1177/107385849700300612

Liu, H., Wu, K., Meusel, P., Seitz, N., Hirzinger, G., Jin, M. H., et al. (2008). Multisensory five-finger dexterous hand: the DLR/HIT hand II. Proc. IEEE/RSJ Int. Conf. Intell. Robots Syst. 3692-3697. doi: 10.1109/IROS.2008.4650624

Mason, C. R., Gomez, J. E., and Ebner, T. J. (2001). Hand synergies during reachto-grasp. J. Neurophysiol. 86, 2896-2910.

Napier, J. (1980). Hands. New York, NY: Pantheon Books.

Oldfield, R. C. (1971). The assessment and analysis of handedness: the Edinburgh inventory. Neuropsychologia 9, 97-113. doi: 10.1016/0028-3932(71)90067-4

Poggio, T., and Bizzi, E. (2004). Generalization in vision and motor control. Nature 431, 768-774. doi: 10.1038/nature03014

Poliakov, A. V., and Schieber, M. H. (1999). Limited functional grouping of neurons in the motor cortex hand area during individuated finger movements: a cluster analysis. J. Neurophysiol. 82, 3488-3505.

Rácz, K., Brown, D., and Valero-Cuevas, F. (2012). An involuntary stereotyical grasp tendency pervades voluntary dynamic multifinger manipulation. J. Neurophysiol. 108, 2896-2911. doi: 10.1152/jn.00297.2012

Raspopovic, S., Capogrosso, M., Petrini, F. M., Bonizzato, M., Rigosa, J., Pino, G. D., et al. (2014). Restoring natural sensory feedback in real-time bidirectional hand prostheses. Sci. Trans. Med. 6, 22ra19. doi: 10.1126/scitranslmed.3006820

Reilly, K. T., and Schieber, M. H. (2003). Incomplete functional subdivision of the human multi-tendon finger muscle flexor digitorum profundus: an electromyographic study. J. Neurophysiol. 90, 2560-2570. doi: 10.1152/jn.00287.2003

Romano, J. M., Hsiao, K., Niemeyer, G., Chitta, S., and Kuchenbecher, K. J. (2011). Human-inspired robotic grasp control with tactile sensing. IEEE Trans. Robot. 27, 1-11. doi: 10.1109/TRO.2011.2162271

Rothling, F., Haschke, R., Steil, J. J., and Ritter, H. (2007). "Platform portable anthropomorphic grasping with the bielefeld 20-DOF shadow and 9-DOF TUM hand," in IEEE/RSJ International Conference on Intelligent Robots and Systems, 2007. IROS 2007 (San Diego, CA), 2951-2956.

Santello, M., Flanders, M., and Soechting, J. F. (1998). Postural hand synergies for tool use. J Neurosci. 18, 10105-10115.

Santello, M., Flanders, M., and Soechting, J. F. (2002). Patterns of hand motion during grasping and the influence of sensory guidance. J. Neurosci. 22, 1426-1435.

Schack, T., and Ritter, H. (2009). The cognitive nature of action-functional links between cognitive psychology, movement science, and robotics. Prog. Brain Res. 174, 231-250. doi: 10.1016/S0079-6123(09)01319-3

Schröder, M., Elbrechter, C., Maycock, J., Haschke, R., Botsch, M., and Ritter, J. H. (2012). "Real-time hand tracking with a color glove for the actuation of anthropomorphic robot hands," in IEEE-RAS International Conference on Humanoid Robots (Humanoids) (Osaka), 262-269.

Semmlow, J. (2001). Biosignal and Biomedical Image Processing. New York, NY: Marcel Dekker, Ink.

Soechting, J. F., and Flanders, M. (1997). Flexibility and repeatability of finger movements during typing: analysis of multiple degrees of freedom. J. Comput. Neurosci. 4, 29-46. doi: 10.1023/A:1008812426305

Steffen, J., Haschke, R., and Ritter, H. (2007). "Experience-based and tactile-driven dynamic grasp control," in IEEE/RSJ International Conference on Intelligent Robots and Systems, 2007. IROS 2007 (San Diego, CA), 2938-2943.

Stockwell, R. A. (1981). "Joints," in Cunningham's Text-Book of Anatomy, ed G. J. Romnes (Oxford: Oxford University Press), 211-264.
Taylor, D., Tillery, S. I. H., and Schwartz, A. B. (2002). Direct cortical control of 3D neuroprosthetic devices. Science 296, 1829-1832. doi: 10.1126/science. 1070291

Thomik, A., Haber, D., and Faisal, A. (2013). Real-time movement prediction for improved control of neuroprosthetics. IEEE/EMBS Conf. Proc. Neural Eng. 6, 625-628. doi: 10.1109/NER.2013.6696012

Tipping, M., and Bishop, C. (1999). Probabilistic principal component analysis. J. R. Stat. Soc. 61, 611-622.

Todorov, E., and Ghahramani, Z. (2004). Analysis of the synergies underlying complex hand manipulation. IEEE Conf. Proc. EMBS 6, 4637-4640. doi: 10.1109/IEMBS.2004.1404285

Tresch, M. C., Cheung, V. C. K., and d'Avella, A. (2006). Matrix factorization algorithms for the identification of muscle synergies: evaluation on simulated and experimental data sets. J. Neurophysiol. 95, 2199-2212. doi: 10.1152/jn.00222.2005

Tubiana, R. (1981). "Architecture and function of the hand," in The Hands, ed R. Tubiana (Philadelphia, PA: Saunders), 19-93.

Valero-Cuevas, F., Venkadesan, M., and Todorov, E. (2009). Structured variability of muscle activations supports the minimal intervention principle of motor control. J. Neurophysiol. 102, 59-68. doi: 10.1152/jn.90324.2008

Velliste, M., Perel, S., Spalding, M. C., Whitford, A. S., and Schwartz, A. B. (2008). Cortical control of a prosthetic arm for self-feeding. Nature 453, 1098-1101. doi: $10.1038 /$ nature 06996

Weiss, E., and Flanders, M. (2004). Muscular and postural synergies of the human hand. J. Neurophysiol. 92, 523-535. doi: 10.1152/jn.01265.2003

Wolpaw, J. R., and McFarland, D. J. (1994). Multichannel EEG-based braincomputer communication. Electroencephalogr. Clin. Neurophysiol. 90, 444-449. doi: 10.1016/0013-4694(94)90135-X

Wolpaw, J. R., and McFarland, D. J. (2004). Control of a two-dimensional movement signal by a noninvasive brain-computer interface in humans. Proc. Natl. Acad. Sci. U.S.A. 101, 17849-17854. doi: 10.1073/pnas.0403 504101

Zhou, P., Lowery, M. M., Englehart, K. B., Huang, H., Li, G., Hargrove, L., et al. (2007). Decoding a new neural machine interface for control of artificial limbs. J. Neurophysiol. 98, 2974-2982. doi: 10.1152/jn.00178.2007

Conflict of Interest Statement: The authors declare that the research was conducted in the absence of any commercial or financial relationships that could be construed as a potential conflict of interest.

Received: 26 October 2014; accepted: 10 February 2015; published online: 26 February 2015.

Citation: Belić JJ and Faisal AA (2015) Decoding of human hand actions to handle missing limbs in neuroprosthetics. Front. Comput. Neurosci. 9:27. doi: 10.3389/fncom. 2015.00027

This article was submitted to the journal Frontiers in Computational Neuroscience. Copyright (c) 2015 Belić and Faisal. This is an open-access article distributed under the terms of the Creative Commons Attribution License (CC BY). The use, distribution or reproduction in other forums is permitted, provided the original author(s) or licensor are credited and that the original publication in this journal is cited, in accordance with accepted academic practice. No use, distribution or reproduction is permitted which does not comply with these terms. 